\title{
Impact of diabetes-related gene polymorphisms on the clinical characteristics of type 2 diabetes Chinese Han population
}

\author{
Jing $\mathrm{Li}^{1, *}$, Jiachen $\mathrm{Wei}^{2, *}$, Pengcheng $\mathrm{Xu}^{3}$, Mengdan Yan ${ }^{1,4}$, Jingjie $\mathrm{Li}^{1,4}$, Zhengshuai \\ Chen ${ }^{1,4}$, Tianbo Jin ${ }^{1,4}$ \\ ${ }^{1}$ Key Laboratory of Resource Biology and Biotechnology in Western China (Northwest University), Ministry of Education, \\ School of Life Sciences, Northwest University, Xi'an, Shaanxi 710069, China \\ ${ }^{2}$ Department of Endocrinology, Xi'an NO.1 Hospital, Xi'an 710002, China \\ ${ }^{3}$ Inner Mongolia Medical University, Inner Mongolia, Hohhot 010010, China \\ ${ }^{4}$ Xi'an Tiangen Precision Medical Institute, Xi'an, Shaanxi, 710075, China \\ * These authors have contributed equally to this work \\ Correspondence to: Tianbo Jin, email: jintianbo@gmail.com \\ Keywords: type2 diabetes, single nucleotide polymorphism, case-control, genetic \\ Received: August 19, $2016 \quad$ Accepted: October 19, $2016 \quad$ Published: November 16, 2016
}

\section{ABSTRACT}

We investigated the correlation between type 2 diabetes (T2D)-related genes and the clinical characteristics of T2D in the Chinese Han population. Our study included 319 patients and 387 controls. Age, gender, clinical features, medications intake and biochemical blood profiles were analyzed. Genotyping was performed on a total of 18 single nucleotide polymorphisms previously reported to be associated with T2D. Our analyses revealed that the CT genotype of ARHGAP22 rs4838605 is associated with T2D risk. Upon analyzing the subjects' clinical characteristics, we found that for rs2811893, the TT genotype correlated with high creatinine levels, while the AA genotype of rs 17045754 and the TT genotype of rs4838605 correlated with elevated triglyceride levels. In addition, the AA genotype of rs17376456 and the TT genotype of rs6214 $(p=0.006)$ correlated with elevated hemoglobin A1c levels. Lastly, those carrying the TT genotype of rs7772697 and the CA genotype of rs3918227 exhibited higher mean body mass index and Cystatin $C$ than controls. Our results showing that the ARHGAP22 gene is associated with an increased risk of T2D, and that seven SNPs in MYSM1, PLXDC2, ARHGAP22 and HS6ST3 promote T2D progression and could help predict the clinical course of T2D in patients at risk.

\section{INTRODUCTION}

Hallmarks of type 2 diabetes (T2D) include insulin resistance and reduced insulin secretion [1-4]. Alteration of glucose metabolism is the primary cause of T2D [5, 6]. In early stages, T2D is often asymptomatic and can remain undetected for several years [7]. According to the International Diabetes Federation (IDF), as of April 2016 415 million people worldwide had diabetes, a number that will rise to 642 million (http://www.diabetesatlas.org/) by 2040.

Previous studies have shown that T2D risk is largely determined by lifestyle habits including active smoking [8], quality of sleep [9], weight loss [10], fruit and vegetable intake [11], and coffee consumption [12]. At the same time, hundreds of studies reported associations between single nucleotide polymorphisms (SNPs) and T2D risk (http://diabetes.diabetesjournals.org). Genetic susceptibility to T2D risk is multifactorial. In the past few years, many additional T2D risk loci have been identified including TCF7L2, FTO, HHEX, SLC30A8, HMG20A, IGF2BP2, MYSM1, TNFSF4, MYT1L, KIAA0825, VEGFA, PLXDC2, CREB5, NOS3, ARHGAP22, IGF1 [13-21]. However, for many of the reports the association between SNPs and T2D risk might have been false-positives or of minor impact in different populations. Indeed, single population studies might not fully explain associations between SNPs of candidate genes and T2D risk.

Early T2D diagnosis can reduce long-term complications, such as diabetic retinopathy, kidney failure, 
cardiovascular disease and limb amputation [22-25]. Unfortunately, screening tests to measure T2D risk are not consistent. The most widely used screening tests include the fasting plasma glucose (FPG) test, glycosylated hemoglobin A1c (HbAlc) test, and the oral glucose tolerance test (OGTT) [26]. We propose that screening methods can be improved by combining analyses of genetic polymorphisms with a lifestyle patterns to predict T2D. In this study, our aim was to investigate the relationship between newly discovered genes associated with T2D susceptibility and the clinical characteristic of T2D in the Chinese Han population.

\section{RESULTS}

\section{Demographic and clinical profile of cases and controls}

The demographic characteristics of the participants are presented in Table 1. The cases and controls were matched by sex, but there was a difference in age between cases and controls $(p=0.021)$, averaging $58.89 \pm 12.01$ and $57.05 \pm 8.25$ years, respectively.

\section{Characteristic of SNPs in the study population}

The basic information of candidate SNPs in our study including gene name, chromosome, minor allele frequency (MAF), and Hardy-Weinberg Equilibrium (HWE) test, appears in Table 2. SNPs at MAF $<0.05$ were removed from association analyses. The HWE agreed with $p>0.05$. However, we identified no significant association between SNPs and T2D risk under the allelic model (Table 2).

\section{Relationships between gene polymorphisms and clinical features}

Genes polymorphisms were also analyzed to establish their associations with clinical features, including gender, age, BMI, duration of diabetes (year), fasting glucose, complications, biochemical criterion, drinking and smoking status, and hypoglycemic drug consumption. We investigated the relationship between candidate gene polymorphisms and the various clinical features listed in patients with T2D. Results for MYSM1, KIAA0825, NOS3, ARHGAP2, and IGF1 were listed in Table 3. For rs2811893, the TT genotype appeared to have high $\mathrm{Cr}$ levels in cases (mean $\pm \mathrm{SD}=71.18 \pm 38.63, p=0.006$ ), compared with the $\mathrm{TC}+\mathrm{CC}$ genotype. The AA genotype of rs 17045754 and the TT genotype of rs4838605 exhibited higher levels of triglycerides (TG) (mean $\pm \mathrm{SD}=7.59 \pm$ 5.17, $p=0.001$ and mean $\pm \mathrm{SD}=2.66 \pm 2.36, p=0.041$, respectively). Moreover, The AA genotype of rs17376456 and the TT genotype of rs6214 are associated with high levels of hemoglobin $\mathrm{A} 1 \mathrm{C}(\mathrm{HbA} 1 \mathrm{C})$ (mean $\pm \mathrm{SD}=9.35 \pm$ $2.38, p=0.039$ and mean $\pm \mathrm{SD}=10.10 \pm 3.34, p=0.006$, respectively). We also found that the TT genotype of rs 7772697 and the CA genotype of rs3918227 had a higher mean $\mathrm{BMI}($ mean $\pm \mathrm{SD}=25.25 \pm 3.48, p=0.028)$ and Cystatin C (Cys-c) $($ mean $\pm \mathrm{SD}=1.02 \pm 0.91, p=0.045)$, respectively (Table 3 ).

\section{Association of genotype and genetic models with T2D risk}

The observed genotypes of the above seven SNPs for cases were compared to those of controls. The genotype frequencies of the seven gene polymorphisms MYSM1 (rs2811893), LEKR1-CCNL1 (rs13064954), KIAA0825 (rs17376456), UST-TAB2 (rs7772697), NOS3 (rs3918227), ARHGAP22 (rs4838605), and IGF1 (rs6214) were shown in Table 4. The rs4838605 genotype frequency distribution in T2D patients was: TT, CT, CC. The CT genotype increased the risk of T2D compared with TT $(\mathrm{CT}$ vs TT: $\mathrm{OR}=1.54,95 \% \mathrm{CI}=$ $1.06-2.24, p=0.003)$. Furthermore, we assumed that the minor allele of each SNP was a risk allele compared to the wild type allele. Four genetic models including logadditive, over-dominant, dominant and recessive were applied to analyze the associations between the SNPs and T2D risk using a logistic regression test in Table 5. We found that the minor allele " $\mathrm{C}$ " of rs 4838605 has an association with increased T2D risk under the overdominant model $(\mathrm{OR}=1.57 ; 95 \% \mathrm{CI}=1.08-2.27$; $p=0.018$ ) (Table 5). In order to assess the associations between SNP haplotypes and T2D risk, a Wald test was performed using an unconditional multivariate regression analysis. However, no positive results were observed (data not shown).

\section{DISCUSSION}

There are several biochemical markers for T2D including Cr, TG, HbA1C and Cys-c levels. An interplay between genetic and environmental factors seems to determine T2D risk. In this study, we selected previously published genetic loci associated with diabetic retinopathy to investigate their relationship with T2D risk and clinical characteristics of T2D in the Chinese Han population. Our results suggest that MYSM1, PLXDC2, ARHGAP22 and HS6ST3 might promote T2D, with ARHGAP22 showing the strongest association with T2D risk $(p=0.018)$. In addition, we found significant correlations between SNPs and clinical features including (1) rs2811893 (MYSM1) T to $\mathrm{C}$ change and Cr levels; (2) A allele in rs13064954 and $\mathrm{C}$ allele in rs4838605 (ARHGAP22) polymorphism and TG levels; (3) T allele in rs6214 (IGF1) and $\mathrm{G}$ allele in rs17376456 (KIAA0825) polymorphism and HbA1c levels; (4) C allele in rs7772697 (unknown) polymorphism and BMI; and (5) A allele in rs3918227 (NOS3) polymorphism and Cys-c levels. 
Table 1: Basic characteristics of diabetes patients and controls

\begin{tabular}{|c|c|c|c|c|}
\hline Characteristics & & Case & Control & $p$-value \\
\hline \multicolumn{5}{|l|}{ Demographic } \\
\hline Number & & 319 & 387 & \\
\hline Gender & & & & 0.258 \\
\hline female & & 143 & 190 & \\
\hline male & & 176 & 197 & \\
\hline Age(year) & $($ mean $\pm \mathrm{SD})$ & $58.89 \pm 12.01$ & $57.05 \pm 8.25$ & $0.021^{*}$ \\
\hline \multicolumn{5}{|l|}{ Clinical } \\
\hline BMI, kg/m² & $($ mean $\pm \mathrm{SD})$ & $24.92 \pm 3.44$ & & \\
\hline Duration of diabetes(year) & $($ mean $\pm \mathrm{SD})$ & $9.37 \pm 7.76$ & & \\
\hline Fasting glucose $(\mathrm{mmol} / \mathrm{L})$ & $($ mean $\pm \mathrm{SD})$ & $9.82 \pm 4.53$ & & \\
\hline \multirow[t]{2}{*}{ Complication } & Negative & 84 & & \\
\hline & Positive & 235 & & \\
\hline \multirow[t]{2}{*}{ Smoker } & Negative & 221 & & \\
\hline & Positive & 98 & & \\
\hline \multirow[t]{2}{*}{ Drinker } & Negative & 266 & & \\
\hline & Positive & 53 & & \\
\hline \multicolumn{5}{|l|}{ Biochemical } \\
\hline $\mathrm{HbA1c}(\mathrm{mmol} / \mathrm{L})$ & $($ mean $\pm \mathrm{SD})$ & $9.25 \pm 2.33$ & & \\
\hline Total cholesterol(mmol/L) & $($ mean $\pm \mathrm{SD})$ & $4.64 \pm 1.27$ & & \\
\hline Triglycerides(mmol/L) & $($ mean $\pm \mathrm{SD})$ & $2.53 \pm 2.21$ & & \\
\hline LDL cholesterol(mmol/L) & $($ mean $\pm \mathrm{SD})$ & $2.78 \pm 0.91$ & & \\
\hline HDL cholesterol(mmol/L) & $($ mean $\pm \mathrm{SD})$ & $1.20 \pm 0.53$ & & \\
\hline $\operatorname{Urea}(\mathrm{mmol} / \mathrm{L})$ & $($ mean $\pm \mathrm{SD})$ & $6.27 \pm 2.38$ & & \\
\hline $\mathrm{Cr}(\mathrm{mmol} / \mathrm{L})$ & $($ mean $\pm \mathrm{SD})$ & $65.47 \pm 28.32$ & & \\
\hline Cys-c(mmol/L) & $($ mean $\pm \mathrm{SD})$ & $0.87 \pm 0.42$ & & \\
\hline GFR(mmol/L) & $($ mean $\pm \mathrm{SD})$ & $123.70 \pm 34.18$ & & \\
\hline $\mathrm{CP}(\mathrm{mmol} / \mathrm{L})$ & $($ mean $\pm \mathrm{SD})$ & $1.40 \pm 1.48$ & & \\
\hline INS(mmol/L) & $($ mean $\pm \mathrm{SD})$ & $18.73 \pm 17.48$ & & \\
\hline $\mathrm{UCRP}(\mathrm{mmol} / \mathrm{L})$ & $($ mean $\pm \mathrm{SD})$ & $0.52 \pm 1.14$ & & \\
\hline \multicolumn{5}{|l|}{ Medications } \\
\hline \multirow[t]{2}{*}{ Hypoglycemic drugs } & Negative & 194 & & \\
\hline & Positive & 125 & & \\
\hline \multirow[t]{2}{*}{ Patients on insulin therapy } & Negative & 156 & & \\
\hline & Positive & 163 & & \\
\hline
\end{tabular}

${ }^{*} p<0.05$ indicates statistical significance;

Continuous variables are expressed as mean $\pm \mathrm{SD}$;

Comparisons between groups were performed using ANOVA for continuous variables and $\mathrm{x}^{2}$ test for categorical variables.

We investigated the association between 18 SNPs and T2D risk. We found that the rs4838605 (ARHGAP22) gene is associated with increased T2D risk $(p=0.018)$, consistent with previous studies. McAuley et al. found that diabetic retinopathy patients with rs4838605 polymorphism have a 1.58- fold increase in T2D risk under the co-dominant model [27]. Yu-Chuen Huang et al. provided the first evidence that $A R H G A P 22$ polymorphisms were involved in endothelial cell angiogenesis [17]. ARHGAP22 encodes for a GTPase protein in the Rho family, which is involved in the signal transduction pathway that regulates endothelial cell capillary tube formation during angiogenesis [28, 29]. Recently, ARHGAP22 levels have been proposed to determine tumor cell movement $[30,31]$ and the 
Table 2: Basic information on candidate SNPs

\begin{tabular}{lcccccccccc}
\hline SNP ID & Genes & Chromosome & $\begin{array}{c}\text { Alleles } \\
\text { A/B }\end{array}$ & $\begin{array}{c}\text { MAF- } \\
\text { case }\end{array}$ & $\begin{array}{c}\text { MAF- } \\
\text { control }\end{array}$ & $\begin{array}{c}\text { HWE test } \\
\boldsymbol{p} \text {-value }\end{array}$ & ORs & 95\%CI & $\begin{array}{c}\boldsymbol{p}^{*} \\
\text { value }\end{array}$ \\
\hline rs3007729 & Unknown & 1 & $\mathrm{~T} / \mathrm{C}$ & $\mathrm{T}$ & $\mathrm{C}$ & 1.000 & 1.23 & 1.00 & 1.53 & 0.052 \\
rs2811893 & MYSM1 & 1 & $\mathrm{C} / \mathrm{T}$ & $\mathrm{C}$ & $\mathrm{T}$ & 0.230 & 0.95 & 0.76 & 1.18 & 0.633 \\
rs1342038 & TNFSF4 & 1 & $\mathrm{G} / \mathrm{A}$ & $\mathrm{G}$ & $\mathrm{A}$ & 0.599 & 0.88 & 0.71 & 1.08 & 0.224 \\
rs10927101 & Unknown & 1 & $\mathrm{C} / \mathrm{A}$ & $\mathrm{C}$ & $\mathrm{A}$ & 0.909 & 1.01 & 0.81 & 1.26 & 0.925 \\
rs10199521 & MYT1L & 2 & $\mathrm{~T} / \mathrm{C}$ & $\mathrm{T}$ & $\mathrm{C}$ & 0.897 & 1.18 & 0.94 & 1.49 & 0.157 \\
rs13064954 & Unknown & 3 & $\mathrm{~A} / \mathrm{G}$ & $\mathrm{A}$ & $\mathrm{G}$ & 0.288 & 0.95 & 0.67 & 1.33 & 0.758 \\
rs17376456 & KIAA0825 & 5 & $\mathrm{G} / \mathrm{A}$ & $\mathrm{G}$ & $\mathrm{A}$ & 0.713 & 0.79 & 0.52 & 1.20 & 0.267 \\
rs3025040 & VEGFA & 6 & $\mathrm{~T} / \mathrm{C}$ & $\mathrm{T}$ & $\mathrm{C}$ & 0.731 & 0.86 & 0.65 & 1.14 & 0.302 \\
rs7772697 & Unknown & 6 & $\mathrm{C} / \mathrm{T}$ & $\mathrm{C}$ & $\mathrm{T}$ & 0.346 & 1.06 & 0.80 & 1.41 & 0.671 \\
rs11765845 & CREB5 & 7 & $\mathrm{~A} / \mathrm{G}$ & $\mathrm{A}$ & $\mathrm{G}$ & 1.000 & 0.98 & 0.77 & 1.24 & 0.872 \\
rs1799983 & NOS3 & 7 & $\mathrm{~T} / \mathrm{G}$ & $\mathrm{T}$ & $\mathrm{G}$ & 0.083 & 1.13 & 0.81 & 1.59 & 0.471 \\
rs3918227 & NOS3 & 7 & $\mathrm{~A} / \mathrm{C}$ & $\mathrm{A}$ & $\mathrm{C}$ & 0.222 & 1.18 & 0.79 & 1.77 & 0.426 \\
rs1571942 & PLXDC2 & 10 & $\mathrm{G} / \mathrm{A}$ & $\mathrm{G}$ & $\mathrm{A}$ & 1.000 & 1.05 & 0.74 & 1.50 & 0.779 \\
rs12219125 & Unknown & 10 & $\mathrm{~T} / \mathrm{G}$ & $\mathrm{T}$ & $\mathrm{G}$ & 1.000 & 1.07 & 0.75 & 1.52 & 0.716 \\
rs4838605 & ARHGAP22 & 10 & $\mathrm{C} / \mathrm{T}$ & $\mathrm{C}$ & $\mathrm{T}$ & 0.396 & 1.24 & 0.89 & 1.74 & 0.207 \\
rs6219 & IGF1 & 12 & $\mathrm{~T} / \mathrm{C}$ & $\mathrm{T}$ & $\mathrm{C}$ & 0.601 & 1.13 & 0.86 & 1.48 & 0.374 \\
rs6214 & IGF1 & 12 & $\mathrm{~T} / \mathrm{C}$ & $\mathrm{T}$ & $\mathrm{C}$ & 0.839 & 0.90 & 0.73 & 1.11 & 0.332 \\
rs10403021 & Unknown & 19 & $\mathrm{~T} / \mathrm{C}$ & $\mathrm{T}$ & $\mathrm{C}$ & 0.706 & 1.14 & 0.91 & 1.44 & 0.264 \\
\hline
\end{tabular}

$\mathrm{MAF}=$ minor allele frequency; HWE $=$ Hardy-Weinberg Equilibrium;

$\mathrm{OR}=$ odds ratio $; 95 \% \mathrm{CI}=95 \%$ confidence interval.

ARHGAP22 gene has been implicated in a novel insulinregulated pathway $[32,33]$. In our study, $A R H G A P 22$ was the only gene associated with T2D risk, which suggests that ARHGAP22 might function as an insulin regulator.

Individuals with high levels of $\mathrm{Cr}, \mathrm{TG}$, Cys-c or $\mathrm{HbA} 1 \mathrm{C}$ are more susceptible to T2D. BMI is also strongly associated with T2D risk [34]. Here, the variant rs17376456 and rs6214 genotypes presented elevated $\mathrm{HbA} 1 \mathrm{c}$ levels and rs2811893 correlated with high $\mathrm{Cr}$ levels. Furthermore, the variant genotypes of rs13064954 and rs4838605 exhibited elevated TG levels. For the rs3918227 polymorphism, Cys-c levels were associated with increased T2D risk and CA genotype. Lastly, the rs7772697 polymorphism correlated with BMI status. These results suggest that the aforementioned SNPs promoted the development and progression of T2D and may help to predict the clinical course of T2D.

Diabetic nephropathy, predominantly due to T2D, is the most frequent cause of renal failure in the United States and in Europe $[35,36]$. Therefore, prevention or delay of diabetic renal disease could improve the life of patients at risk [37]. Glomerular filtration rate (GFR) is the best overall index of renal function. Cys-C has been proposed as a reliable serum marker for GFR [38-41]. In addition, Oddoze et al. found that serum $\mathrm{Cr}$ can estimate the GFR in diabetic patients [42]. In this study, we found that rs2811893 in the MYSM1 gene and rs3918227 in the NOS3 polymorphism present elevated levels of $\mathrm{Cr}$ and Cys-C, respectively. We concluded that MYSM1 and polymorphisms in the NOS3 gene might contribute to T2D risk in the Chinese Han population.

Although our study suffered from some limitations, such as a relatively small sample size and a lack of data on the lifestyle habits of patients (e.g., active smoking, quality of sleep, weight loss, and diet), our results indicated that seven SNPs in MYSM1, PLXDC2, ARHGAP22, and HS6ST3, increase T2D risk in the Chinese Han population. Functional studies on gene-gene and gene-environment interactions and clinical studies with larger sample sizes could further substantiate the impact of these gene polymorphisms on T2D. 
Table 3: The associations between genetic polymorphisms and clinical characteristics of T2D

\begin{tabular}{|c|c|c|c|c|c|c|c|c|c|c|c|c|}
\hline \multirow{2}{*}{ SNP } & \multirow{2}{*}{ genotype } & \multirow{2}{*}{$\mathbf{n}$} & \multicolumn{2}{|c|}{ HbA1c } & \multicolumn{2}{|l|}{ TG } & \multicolumn{2}{|l|}{$\mathrm{Cr}$} & \multicolumn{2}{|c|}{ Cys-c } & \multicolumn{2}{|l|}{ BMI } \\
\hline & & & mean $\pm \mathrm{SD}$ & $p^{*}$ & mean $\pm \mathrm{SD}$ & $p^{*}$ & mean $\pm \mathrm{SD}$ & $p^{*}$ & mean $\pm \mathrm{SD}$ & $p^{*}$ & mean $\pm \mathrm{SD}$ & $p^{*}$ \\
\hline \multirow[t]{3}{*}{ rs2811893(MYSM1) } & $\mathrm{TT}$ & 130 & $9.56 \pm 2.81$ & 0.083 & $2.53 \pm 2.20$ & 0.906 & $71.18 \pm 38.63$ & $0.006^{*}$ & $0.87 \pm 0.26$ & 0.975 & $24.79 \pm 3.59$ & 0.495 \\
\hline & CT & 148 & $9.15 \pm 1.98$ & & $2.51 \pm 2.19$ & & $61.95 \pm 18.39$ & & $0.87 \pm 0.56$ & & $24.90 \pm 3.36$ & \\
\hline & $\mathrm{CC}$ & 40 & $8.68 \pm 1.66$ & & $2.68 \pm 2.37$ & & $11.93 \pm 1.89$ & & $0.86 \pm 0.17$ & & $25.52 \pm 3.24$ & \\
\hline \multirow[t]{3}{*}{ rs13064954(Unknown) } & AA & 4 & $10.95 \pm 2.03$ & 0.343 & $7.59 \pm 5.17$ & $0.001^{*}$ & $66.47 \pm 21.70$ & 0.584 & $0.85 \pm 0.20$ & 0.615 & $25.27 \pm 3.94$ & 0.555 \\
\hline & GG & 257 & $9.22 \pm 2.40$ & & $2.53 \pm 2.20$ & & $64.67 \pm 27.78$ & & $0.85 \pm 0.23$ & & $24.82 \pm 3.52$ & \\
\hline & $\mathrm{AG}$ & 58 & $9.25 \pm 1.99$ & & $2.22 \pm 1.51$ & & $68.94 \pm 31.10$ & & $0.97 \pm 0.84$ & & $25.35 \pm 3.06$ & \\
\hline \multirow[t]{2}{*}{ rs17376456(KIAA0825) } & AA & 280 & $9.35 \pm 2.38$ & $0.039^{*}$ & $2.56 \pm 2.91$ & 0.6 & $65.56 \pm 29.67$ & 0.88 & $0.87 \pm 0.44$ & 0.526 & $24.92 \pm 3.49$ & 0.884 \\
\hline & GA & 39 & $8.53 \pm 1.78$ & & $2.36 \pm 1.49$ & & $64.83 \pm 15.70$ & & $0.83 \pm 0.20$ & & $25.00 \pm 3.44$ & \\
\hline \multirow[t]{3}{*}{ rs7772697(Unknown) } & $\mathrm{TT}$ & 223 & $9.18 \pm 2.40$ & 0.455 & $2.61 \pm 2.38$ & 0.584 & $65.88 \pm 29.90$ & 0.916 & $0.88 \pm 0.48$ & 0.779 & $25.25 \pm 3.48$ & $0.028^{*}$ \\
\hline & $\mathrm{CT}$ & 85 & $9.51 \pm 2.26$ & & $2.39 \pm 1.83$ & & $64.48 \pm 25.11$ & & $0.85 \pm 0.19$ & & $24.09 \pm 3.34$ & \\
\hline & $\mathrm{CC}$ & 11 & $8.85 \pm 1.23$ & & $2.08 \pm 0.90$ & & $63.29 \pm 18.46$ & & $0.83 \pm 0.30$ & & $24.69 \pm 2.28$ & \\
\hline \multirow[t]{2}{*}{ rs3918227(NOS3) } & $\mathrm{CA}$ & 49 & $8.96 \pm 1.74$ & 0.338 & $2.00 \pm 1.07$ & 0.117 & $63.85 \pm 18.03$ & 0.664 & $1.02 \pm 0.91$ & $0.045^{k}$ & $25.09 \pm 3.53$ & 0.075 \\
\hline & $\mathrm{CC}$ & 270 & $9.31 \pm 2.42$ & & $2.63 \pm 2.35$ & & $65.76 \pm 29.82$ & & $0.84 \pm 0.23$ & & $24.89 \pm 3.43$ & \\
\hline \multirow[t]{2}{*}{ rs4838605(ARHGAP22) } & TT & 243 & $9.34 \pm 2.43$ & 0.216 & $2.66 \pm 2.36$ & $0.041^{*}$ & $65.77 \pm 23.91$ & 0.737 & $0.89 \pm 0.46$ & 0.168 & $24.95 \pm 3.28$ & 0.777 \\
\hline & $\mathrm{TC}$ & 76 & $8.97 \pm 1.97$ & & $2.13 \pm 1.56$ & & $64.51 \pm 39.42$ & & $0.81 \pm 0.25$ & & $24.82 \pm 3.92$ & \\
\hline \multirow[t]{3}{*}{ rs6214(IGF1) } & TT & 72 & $10.10 \pm 3.34$ & $0.006^{*}$ & $2.51 \pm 2.36$ & 0.155 & $61.03 \pm 15.20$ & 0.319 & $0.85 \pm 0.27$ & 0.571 & $25.10 \pm 3.43$ & 0.803 \\
\hline & $\mathrm{CC}$ & 105 & $8.98 \pm 1.86$ & & $2.22 \pm 1.60$ & & $66.57 \pm 26.55$ & & $0.90 \pm 0.63$ & & $24.76 \pm 3.51$ & \\
\hline & $\mathrm{TC}$ & 142 & $9.03 \pm 1.91$ & & $2.78 \pm 2.48$ & & $66.90 \pm 34.05$ & & $0.85 \pm 0.26$ & & $24.96 \pm 3.41$ & \\
\hline
\end{tabular}

$\mathrm{HbA1C}=$ glycosylated hemoglobin $\mathrm{A} 1 \mathrm{c} ; \mathrm{TG}=$ triglyceride $\mathrm{Cr}=$ creatinine; Cys-c = cystatin $\mathrm{C} ; \mathrm{BMI}=$ body mass index;

$\mathrm{OR}=$ odds ratio; $95 \% \mathrm{CI}=95 \%$ confidence interval;

${ }^{*} p \leq 0.05$ indicates statistical significance.

Table 4: The polymorphisms of genotype model in the cases and controls and the associations with T2D risk (adjust by age and gender)

\begin{tabular}{|c|c|c|c|c|c|}
\hline SNP & Genotype & Control & Case & OR $(95 \%$ CI $)$ & $p$-value \\
\hline \multirow[t]{3}{*}{ rs2811893 } & $\mathrm{T} / \mathrm{T}$ & $159(41.1 \%)$ & $130(40.9 \%)$ & 1 & 0.470 \\
\hline & $\mathrm{C} / \mathrm{T}$ & $169(43.7 \%)$ & $148(46.5 \%)$ & $1.08(0.78-1.48)$ & \\
\hline & $\mathrm{C} / \mathrm{C}$ & $59(15.2 \%)$ & $40(12.6 \%)$ & $0.81(0.51-1.29)$ & \\
\hline \multirow[t]{3}{*}{ rs13064954 } & $\mathrm{G} / \mathrm{G}$ & $305(78.8 \%)$ & $257(80.6 \%)$ & 1 & 0.420 \\
\hline & G/A & $80(20.7 \%)$ & $58(18.2 \%)$ & $0.87(0.60-1.27)$ & \\
\hline & $\mathrm{A} / \mathrm{A}$ & $2(0.5 \%)$ & $4(1.2 \%)$ & $2.46(0.44-13.64)$ & \\
\hline \multirow[t]{3}{*}{ rs 17376456} & $\mathrm{~A} / \mathrm{A}$ & $329(85 \%)$ & $280(87.8 \%)$ & 1 & 0.310 \\
\hline & $\mathrm{G} / \mathrm{A}$ & $57(14.7 \%)$ & $39(12.2 \%)$ & $0.79(0.51-1.23)$ & \\
\hline & $\mathrm{G} / \mathrm{G}$ & $1(0.3 \%)$ & $0(0 \%)$ & $0.00(0.00-\mathrm{NA})$ & \\
\hline \multirow[t]{3}{*}{ rs7772697 } & $\mathrm{T} / \mathrm{T}$ & $270(70 \%)$ & $223(69.9 \%)$ & 1 & 0.360 \\
\hline & $\mathrm{C} / \mathrm{T}$ & $109(28.2 \%)$ & $85(26.6 \%)$ & $0.92(0.66-1.29)$ & \\
\hline & $\mathrm{T} / \mathrm{T}$ & $7(1.8 \%)$ & $11(3.5 \%)$ & $1.88(0.71-4.97)$ & \\
\hline \multirow[t]{3}{*}{ rs3918227 } & $\mathrm{C} / \mathrm{C}$ & $339(87.6 \%)$ & $270(84.6 \%)$ & 1 & 0.046 \\
\hline & $\mathrm{C} / \mathrm{A}$ & $45(11.6 \%)$ & $49(15.4 \%)$ & $1.41(0.91-2.20)$ & \\
\hline & $\mathrm{A} / \mathrm{A}$ & $3(0.8 \%)$ & $0(0 \%)$ & $0.00(0.00-\mathrm{NA})$ & \\
\hline \multirow[t]{3}{*}{ rs4838605 } & $\mathrm{T} / \mathrm{T}$ & $316(81.7 \%)$ & $243(76.2 \%)$ & 1 & $0.003^{*}$ \\
\hline & $\mathrm{C} / \mathrm{T}$ & $66(17.1 \%)$ & $76(23.8 \%)$ & $1.54(1.06-2.24)$ & \\
\hline & $\mathrm{C} / \mathrm{C}$ & $5(1.3 \%)$ & $0(0 \%)$ & $0.00(0.00-\mathrm{NA})$ & \\
\hline \multirow[t]{3}{*}{ rs6214 } & $\mathrm{C} / \mathrm{C}$ & $108(27.9 \%)$ & $105(32.9 \%)$ & 1 & 0.310 \\
\hline & $\mathrm{C} / \mathrm{T}$ & $191(49.4 \%)$ & $142(44.5 \%)$ & $0.76(0.54-1.08)$ & \\
\hline & $\mathrm{T} / \mathrm{T}$ & $88(22.7 \%)$ & $72(22.6 \%)$ & $0.84(0.56-1.28)$ & \\
\hline
\end{tabular}

$\mathrm{CI}=$ confidence interval; $\mathrm{OR}=$ odds ratio; $\mathrm{SNP}=$ single nucleotide polymorphism;

${ }^{*} p \leq 0.05$ indicates statistical significance. 
Table 5: Single-SNP analysis and in different genetic models after adjusted by age \& gender

\begin{tabular}{|c|c|c|c|c|c|c|c|c|c|c|c|c|c|}
\hline \multirow{2}{*}{ SNP } & \multirow{2}{*}{$\begin{array}{l}\text { Minor } \\
\text { Allele }\end{array}$} & \multicolumn{3}{|c|}{ Dominant model } & \multicolumn{3}{|c|}{ Recessive model } & \multicolumn{3}{|c|}{ Over-dominant model } & \multicolumn{3}{|c|}{ Log-additive model } \\
\hline & & OR & $95 \%$ CI & $p$ & OR & $95 \% \mathrm{CI}$ & $p$ & OR & $95 \% \mathrm{CI}$ & $p$ & OR & $95 \%$ CI & $p$ \\
\hline rs2811893 & $\mathrm{C}$ & 1.01 & $0.74 \quad 1.36$ & 0.970 & 0.78 & 0.5 & 0.250 & 1.14 & $0.84 \quad 1.53$ & 0.400 & 0.94 & $0.76 \quad 1.17$ & 0.580 \\
\hline rs13064954 & A & 0.91 & $0.63 \quad 1.32$ & 0.610 & 2.53 & $0.46 \quad 14$ & 0.270 & 0.86 & 0.591 .26 & 0.440 & 0.96 & $0.68 \quad 1.36$ & 0.800 \\
\hline rs17376456 & G & 0.78 & $0.5 \quad 1.21$ & 0.260 & NA & NA NA & NA & 0.8 & $0.51 \quad 1.24$ & 0.310 & 0.77 & $0.5 \quad 1.18$ & 0.230 \\
\hline rs7772697 & $\mathrm{C}$ & 0.98 & $0.7 \quad 1.35$ & 0.880 & 1.93 & $0.73 \quad 5.06$ & 0.180 & 0.9 & $0.64 \quad 1.26$ & 0.530 & 1.04 & $0.78 \quad 1.39$ & 0.780 \\
\hline rs3918227 & A & 1.32 & 0.852 .03 & 0.210 & NA & NA NA & NA & 1.43 & $0.92 \quad 2.22$ & 0.110 & 1.21 & $0.8 \quad 1.83$ & 0.370 \\
\hline rs4838605 & $\mathrm{C}$ & 1.43 & 0.992 .06 & 0.056 & NA & NA NA & NA & 1.57 & $1.08 \quad 2.27$ & $0.018^{*}$ & 1.28 & $0.91 \quad 1.82$ & 0.160 \\
\hline rs6214 & $\mathrm{T}$ & 0.79 & $\begin{array}{ll}0.57 & 1.09\end{array}$ & 0.150 & 1 & $0.7 \quad 1.42$ & 0.980 & 0.82 & $0.61 \quad 1.1$ & 0.190 & 0.91 & $0.74 \quad 1.11$ & 0.350 \\
\hline
\end{tabular}

\section{MATERIALS AND METHODS}

\section{Patients and samples}

Our sample consisted of 706 Chinese Han individuals: 319 patients with T2D and 387 controls with normal glucose tolerance (NGT). These participants were recruited between August 2010 and December 2014 from clinics at the department of Endocrinology, Xi'an NO.1 Hospital, Xi'an, China. The key inclusion criteria of case subjects by the World Health Organization in 2010 [43] were: (1) T2D diagnosis by a qualified respiratory physician; (2) impaired glucose tolerance (IGT) [2-h values in the oral glucose tolerance test (OGTT) exceed $199 \mathrm{mg}$ / dl (11.0mmol/L); (3) hemoglobin A1C (HbA1C) test to diagnose diabetes, with a threshold of $\geq 6.5 \%$, and (4) fasting plasma glucose levels of at least $126 \mathrm{mg} / \mathrm{dl}$ ( $7 \mathrm{mmol} / \mathrm{L}$ in SI units). Individuals taking medication that affects glucose metabolism were excluded. Eligible controls were Chinese Han individuals residing in Northwest China. In addition, all patients gave informed consent and completed a personal interview. The participants provided information about anthropometric characteristics including height and weight to determine body mass index (BMI), as well as, age, sex, family history of diabetes and living habits. Midstream urine samples were collected to assess albumin and creatinine (Cr) levels. All participants consented to venipuncture for DNA and biochemical tests. The study was approved by the First Affiliated Hospital of Xi'an Jiaotong University.

\section{SNP selection and genotyping}

We genotyped a total of 18 SNPs previously reported to be associated with T2D. MYSM1 (rs2811893),
KIAA0825 (rs17376456), PLXDC2 (rs1571942), rs12219125, and ARHGAP22 (rs4838605) were selected from a GWAS concerning T2D in the Taiwanese population [17]. SNPs rs3007729, rs1342038, rs10927101, rs10199521, rs13064954, rs7772697, rs11765845, and rs10403021 were identified from a replication association study of diabetic retinopathy [18]. Finally, rs3025040, rs1799983, rs3918227, rs6219, and rs6214 were chosen from other T2D studies [19-21]. The above SNPs were identified by dbSNP (http://www.ncbi.nlm.nih.gov/ projects/SNP). The MAF of all SNPs was $>5 \%$ in the HapMap of the Chinese Han Beijing (CHB) population. The SNPs were genotyped using Sequenom MassARRAY RS1000 (Sequenom, Inc.) containing: PCR amplification assay, designed primers and probes, purification by Shrimp Alkaline Phosphatase (SAP), followed by addition of primers and extension of the special basic group, followed by stimulation cocrystallization combining sample analyte and chip substrates. Primers were designed for each SNP using Primer3 (http://frodo.wi.mit.edu).

\section{Statistical analysis}

All demographic parameters are presented as mean \pm standard deviation (mean $\pm \mathrm{SD}$ ), unless otherwise specified. Comparisons of participants' characteristics were performed using an independent sample $t$-test (using Levene's test for equality of variances) for continuous variables and an additional Chi-square test for categorical variables. All populations also underwent testing for Hardy-Weinberg equilibrium (HWE). We used the PLINK software package (version 1.07) to assess genetic data. A multivariate logistic regression model, adjusting for age and gender, was used to estimate risk associations between genotypes and $\mathrm{T} 2 \mathrm{D}$ by computing the odds ratio (OR) and $95 \%$ confidence intervals ( $95 \%$ CIs). To investigate potential models of inheritance, the log-additive, over- 
dominant, dominant and recessive genotypic models were applied.

\section{ACKNOWLEDGMENTS}

The authors wish to thank participants in the study who made this work possible. We would also like to thank the clinicians and other hospital staff who contributed to the blood sample and data collection for this study. This work was supported by Opening Foundation of Key Laboratory of Resource Biology and Biotechnology in Western China (Northwest University), Ministry of Education.

\section{CONFLICTS OF INTEREST}

No conflicts of interest to declare.

\section{REFERENCES}

1. Fukushima M, Suzuki H, Seino Y. Insulin secretion capacity in the development from normal glucose tolerance to type 2 diabetes. Diabetes research and clinical practice. 2004; 66:S37-43.

2. Kahn CR, Vicent D, Doria A. Genetics of non-insulindependent (type-II) diabetes mellitus. Annual review of medicine. 1996; 47:509-531.

3. Lehtovirta M, Kaprio J, Forsblom C, Eriksson J, Tuomilehto J, Groop L. Insulin sensitivity and insulin secretion in monozygotic and dizygotic twins. Diabetologia. 2000; 43:285-293.

4. Florez JC, Hirschhorn J, Altshuler D. The inherited basis of diabetes mellitus: implications for the genetic analysis of complex traits. Annual review of genomics and human genetics. 2003; 4:257-291.

5. Brownlee M. Biochemistry and molecular cell biology of diabetic complications. Nature. 2001; 414:813-820.

6. Jerneld B, Algvere P. Relationship of duration and onset of diabetes to prevalence of diabetic retinopathy. American journal of ophthalmology. 1986; 102:431-437.

7. Harris MI, Klein R, Welborn TA, Knuiman MW. Onset of NIDDM occurs at least 4-7 yr before clinical diagnosis. Diabetes care. 1992; 15:815-819.

8. Willi C, Bodenmann P, Ghali WA, Faris PD, Cornuz J. Active smoking and the risk of type 2 diabetes: A systematic review and meta-analysis. JAMA. 2007; 298:2654-2664.

9. Cappuccio FP, D'Elia L, Strazzullo P, Miller MA. Quantity and quality of sleep and incidence of type 2 diabetes: a systematic review and meta-analysis. Diabetes care. 2010; 33:414-420.

10. Anderson JW, Kendall CW, Jenkins DJ. Importance of weight management in type 2 diabetes: review with metaanalysis of clinical studies. Journal of the American College of Nutrition. 2003; 22:331-339.
11. Carter P, Gray LJ, Troughton J, Khunti K, Davies MJ. Fruit and vegetable intake and incidence of type 2 diabetes mellitus: systematic review and meta-analysis. BMJ. 2010; 341 .

12. van Dam RM, Hu FB. Coffee consumption and risk of type 2 diabetes: A systematic review. JAMA. 2005; 294:97-104.

13. Adeyemo AA, Tekola-Ayele F, Doumatey AP, Bentley AR, Chen G, Huang H, Zhou J, Shriner D, Fasanmade O, Okafor G, Eghan B, Jr., Agyenim-Boateng K, Adeleye $\mathrm{J}$, et al. Evaluation of Genome Wide Association Study Associated Type 2 Diabetes Susceptibility Loci in Sub Saharan Africans. Front Genet. 2015; 6:335.

14. Sladek R, Rocheleau G, Rung J, Dina C, Shen L, Serre D, Boutin P, Vincent D, Belisle A, Hadjadj S, Balkau B, Heude $B$, Charpentier G, et al. A genome-wide association study identifies novel risk loci for type 2 diabetes. Nature. 2007; 445:881-885.

15. Scott LJ, Mohlke KL, Bonnycastle LL, Willer CJ, Li Y, Duren WL, Erdos MR, Stringham HM, Chines PS, Jackson AU, Prokunina-Olsson L, Ding CJ, Swift AJ, et al. A genome-wide association study of type 2 diabetes in Finns detects multiple susceptibility variants. Science (New York, NY). 2007; 316:1341-1345.

16. Zeggini E, Weedon MN, Lindgren CM, Frayling TM, Elliott KS, Lango H, Timpson NJ, Perry JR, Rayner NW, Freathy RM, Barrett JC, Shields B, Morris AP, et al. Replication of genome-wide association signals in UK samples reveals risk loci for type 2 diabetes. Science (New York, NY). 2007; 316:1336-1341.

17. Huang YC, Lin JM, Lin HJ, Chen CC, Chen SY, Tsai $\mathrm{CH}$, Tsai FJ. Genome-wide association study of diabetic retinopathy in a Taiwanese population. Ophthalmology. 2011; 118:642-648.

18. McAuley AK, Wang JJ, Dirani M, Connell PP, Lamoureux E, Hewitt AW. Replication of genetic loci implicated in diabetic retinopathy. Investigative ophthalmology \& visual science. 2014; 55:1666-1671.

19. Ferguson JF, Phillips CM, McMonagle J, Perez-Martinez P, Shaw DI, Lovegrove JA, Helal O, Defoort C, Gjelstad IM, Drevon CA, Blaak EE, Saris WH, Leszczynska-Golabek I, et al. NOS3 gene polymorphisms are associated with risk markers of cardiovascular disease, and interact with omega-3 polyunsaturated fatty acids. Atherosclerosis. 2010; 211:539-544.

20. Zhang X, Sun Z, Jiang H, Song X. Relationship between single nucleotide polymorphisms in the 3'-untranslated region of the vascular endothelial growth factor gene and susceptibility to diabetic peripheral neuropathy in China. Archives of medical science. 2014; 10:1028-1034.

21. Meyer S, Schaefer S, Ivan D, Stolk L, Arp P, Uitterlinden AG, Nawroth PP, Plöckinger U, Stalla GK, Tuschy U, Weber MM, Weise A, Pfützner A, Kann PH. Growth hormone dose in growth hormone-deficient adults is not associated with IGF-1 gene polymorphisms. Pharmacogenomics. 2009; 10:293-302. 
22. Retinopathy Progression in Type 2 Diabetes. New England Journal of Medicine. 2010; 363:2171-2174.

23. Boronat M, Garcia-Canton C, Quevedo V, Lorenzo DL, Lopez-Rios L, Batista F, Riano M, Saavedra P, Checa MD. Non-albuminuric renal disease among subjects with advanced stages of chronic kidney failure related to type 2 diabetes mellitus. Renal failure. 2014; 36:166-170.

24. Laakso M. Hyperglycemia and cardiovascular disease in type 2 diabetes. Diabetes. 1999; 48:937-942.

25. Jeffcoate WJ, Harding KG. Diabetic foot ulcers. The Lancet. 2003; 361:1545-1551.

26. Bennett CM, Guo M, Dharmage SC. $\operatorname{HbA}(1 \mathrm{c})$ as a screening tool for detection of Type 2 diabetes: a systematic review. Diabetic medicine. 2007; 24:333-343.

27. McAuley A. (2014). Biomarkers in diabetic retinopathy: genetic and proteomic profiling.

28. Katoh M, Katoh M. Identification and characterization of ARHGAP24 and ARHGAP25 genes in silico. International journal of molecular medicine. 2004; 14:333-338.

29. Katoh M, Katoh M. Characterization of human ARHGAP10 gene in silico. International journal of oncology. 2004; 25:1201-1206.

30. Sanz-Moreno V, Gadea G, Ahn J, Paterson H, Marra P, Pinner S, Sahai E, Marshall CJ. Rac activation and inactivation control plasticity of tumor cell movement. Cell. 2008; 135:510-523.

31. Croft DR, Olson MF. Regulating the conversion between rounded and elongated modes of cancer cell movement. Cancer cell. 2008; 14:349-351.

32. Larance M, Rowland AF, Hoehn KL, Humphreys DT, Preiss T, Guilhaus M, James DE. Global phosphoproteomics identifies a major role for AKT and 14-3-3 in regulating EDC3. Molecular \& cellular proteomics. 2010; 9:682-694.

33. Rowland AF, Larance $M$, Hughes WE, James DE. Identification of RhoGAP22 as an Akt-dependent regulator of cell motility in response to insulin. Molecular and cellular biology. 2011; 31:4789-4800.

34. Organization WH. Obesity: preventing and managing the global epidemic. Report of a WHO consultation. World
Health Organization technical report series. 2000; 894:ixii, 1-253.

35. Ritz E, Rychlik I, Locatelli F, Halimi S. End-stage renal failure in type 2 diabetes: A medical catastrophe of worldwide dimensions. American journal of kidney diseases. 1999; 34:795-808.

36. Ismail N, Becker B, Strzelczyk P, Ritz E. Renal disease and hypertension in non-insulin-dependent diabetes mellitus. Kidney international. 1999; 55:1-28.

37. Mogensen CE, Keane WF, Bennett PH, Jerums G, Parving HH, Passa P, Steffes MW, Striker GE, Viberti GC. Prevention of diabetic renal disease with special reference to microalbuminuria. Lancet (London, England). 1995; 346:1080-1084.

38. Finney H, Newman DJ, Price CP. Adult reference ranges for serum cystatin $\mathrm{C}$, creatinine and predicted creatinine clearance. Annals of clinical biochemistry. 2000; 37:49-59.

39. Coll E, Botey A, Alvarez L, Poch E, Quinto L, Saurina A, Vera M, Piera C, Darnell A. Serum cystatin C as a new marker for noninvasive estimation of glomerular filtration rate and as a marker for early renal impairment. American journal of kidney diseases. 2000; 36:29-34.

40. Newman DJ, Thakkar H, Edwards RG, Wilkie M, White T, Grubb AO, Price CP. Serum cystatin C measured by automated immunoassay: a more sensitive marker of changes in GFR than serum creatinine. Kidney international. 1995; 47:312-318.

41. Mussap M, Dalla Vestra M, Fioretto P, Saller A, Varagnolo M, Nosadini R, Plebani M. Cystatin C is a more sensitive marker than creatinine for the estimation of GFR in type 2 diabetic patients. Kidney international. 2002; 61:1453-1461.

42. Oddoze C, Morange S, Portugal H, Berland Y, Dussol B. Cystatin $\mathrm{C}$ is not more sensitive than creatinine for detecting early renal impairment in patients with diabetes. American journal of kidney diseases. 2001; 38:310-316.

43. Diabetes mellitus. Report of a WHO Study Group. World Health Organization technical report series. 1985; 727:1-113. 